Gut and Liver, Vol. 10, No. 4, July 2016, pp. 495-496

\title{
Towards Steroid-Free Immunosuppression after Liver Transplantation
}

\author{
Victor M. Zaydfudim ${ }^{1}$ and Shawn J. Pelletier ${ }^{2}$ \\ ${ }^{1}$ Section of Hepatobiliary and Pancreatic Surgery, Department of Surgery, and ${ }^{2}$ Charles 0 . Strickler Transplant Center, University of Virginia, \\ Charlottesville, VA, USA
}

See "Efficacy and Safety of a Steroid-Free Immunosuppressive Regimen after Liver Transplantation for Hepatocellular Carcinoma” by Qiang Wei, et al. on page 604, Vol. 10. No. 4, 2016

The deleterious long-term side effects and metabolic complications of corticosteroids have inspired initial clinical investigation of corticosteroid withdrawal and avoidance protocols in liver transplantation. The original studies in the 1990s focused on the safety of steroid withdrawal in patients without hepatocellular carcinoma (HCC). ${ }^{1,2}$ Subsequent clinical trials suggested noninferiority of steroid withdrawal and steroid avoidance regimens after liver transplantation in long-term graft function and patient specific outcomes, including survival. ${ }^{3-5}$ Recent trials included patients with viral etiologies of chronic liver disease (both hepatitis B and hepatitis C) and HCC, however, data evaluating impact of steroid-free regimens on recurrence in patients transplanted for HCC are less robust. A few single-institution retrospective cohort studies suggested greater tumor recurrence and possibly worse survival in patients with higher dose steroid regimens, ${ }^{6,7}$ however convincing prospective data are insufficient.

The current retrospective cohort study by Wei et al., ${ }^{8}$ examines both posttransplant complications and tumor recurrence in patients with hepatitis B virus (HBV) cirrhosis and HCC. All patients had induction with methylprednisolone; patients in the "steroid-free" group also received basiliximab, while patients in the control group had a 3-month prednisolone taper without basiliximab. All patients in both groups had the same two drug long-term immunosuppression regimen. Importantly, all patients received hepatitis B specific antiviral therapy before and after transplantation. The steroid-free protocol appears to be a paradigm change in this group's practice in 2009 and a 1:2 match control group is selected from noncontemporaneous liver transplant recipients preceding the study group. A few tumor specific metrics in this study are notable. Average pretransplant $\alpha$-fetoprotein exceeded 2,000 and $\geq 50 \%$ of patients in both steroid-free and control groups had HCC exceeding Milan criteria. Also, while average tumor size was comparable, average tumor number was significantly higher in the earlier patient group managed with a 3-month steroid taper rather than steroid-free protocol.

Posttransplant complications between two groups are similar. While the exact follow-up time for each group is unclear and is theoretically longer for patients managed with 3-month prednisolone taper, proportions of acute rejection, hypertension, new-onset diabetes mellitus, hyperlipidemia, infectious complications, as well as early renal and allograft dysfunction, are similar in both patient groups. Proportion of patients with HBV recurrence is lower in the more contemporaneous steroid-free patient cohort. Survival outcomes are stratified by steroid use and Milan criteria. The authors suggest greater survival among patients within Milan managed with steroid-free protocol, however, lack of direct tumor biology comparisons ( $\alpha$-fetoprotein, tumor size, and tumor number) for these two patient subgroups and noncontemporaneous controls limit direct comparison to steroid-taper patients within Milan criteria.

This study adds another cobblestone on the path towards safe and effective steroid-free immunosuppression in liver transplant recipients. Current data support clinical efficacy of steroid limiting protocols without increased risk of graft loss or dysfunction after liver transplantation. Potentially diminished chance of HBV recurrence with steroid-free regimens is encouraging and tumor-specific survival data requires further investigation. As long-term survival after liver transplantation continues to improve, minimization of steroids is important. In addition to the undesirable metabolic side-effects, long-term steroid use has

Correspondence to: Victor M. Zaydfudim

Department of Surgery, Box 800709, University of Virginia, Charlottesville, VA 22908-0709, USA

Tel: +1-434-924-2839, Fax: +1-434-982-4778, E-mail: vz8h@virginia.edu

pISSN 1976-2283 eISSN 2005-1212 http://dx.doi.org/10.5009/gnl16204

(a) This is an Open Access article distributed under the terms of the Creative Commons Attribution Non-Commercial License (http://creativecommons.org/licenses/by-nc/4.0) which permits unrestricted non-commercial use, distribution, and reproduction in any medium, provided the original work is properly cited. 
been associated with worse health-related quality of life and psychologic disorders in liver transplant recipients. ${ }^{9}{ }^{90}$ Proliferation of steroid withdrawal, steroid avoidance, and steroid-free immunosuppression protocols should improve long-term patient centered outcomes after liver transplantation.

\section{CONFLICTS OF INTEREST}

No potential conflict of interest relevant to this article was reported.

\section{REFERENCES}

1. Padbury RT, Gunson BK, Dousset B, et al. Steroid withdrawal from long-term immunosuppression in liver allograft recipients. Transplantation 1993;55:789-794.

2. Punch JD, Shieck VL, Campbell DA, Bromberg JS, Turcotte JG, Merion RM. Corticosteroid withdrawal after liver transplantation. Surgery 1995;118:783-786.

3. De Carlis L, Belli LS, Rondinara GF, et al. Early steroid withdrawal in liver transplant patients: final report of a prospective randomized trial. Transplant Proc 1997;29:539-542.

4. Pelletier SJ, Nadig SN, Lee DD, et al. A prospective, randomized trial of complete avoidance of steroids in liver transplantation with follow-up of over 7 years. HPB (Oxford) 2013;15:286-293.
5. Lerut JP, Pinheiro RS, Lai Q, et al. Is minimal, [almost] steroid-free immunosuppression a safe approach in adult liver transplantation? Long-term outcome of a prospective, double blind, placebocontrolled, randomized, investigator-driven study. Ann Surg 2014; 260:886-891.

6. Miyagi S, Kawagishi N, Sekiguchi S, et al. The relationship between recurrences and immunosuppression on living donor liver transplantation for hepatocellular carcinoma. Transplant Proc 2012;44:797-801.

7. Xing T, Huang L, Yu Z, Zhong L, Wang S, Peng Z. Comparison of steroid-free immunosuppression and standard immunosuppression for liver transplant patients with hepatocellular carcinoma. PLoS One 2013;8:e71251.

8. Wei Q, Xu X, Wang C, et al. Efficacy and safety of a steroid-free immunosuppressive regimen after liver transplantation for hepatocellular carcinoma. Gut Liver 2016;10:604-610.

9. Zaydfudim V, Feurer ID, Landman MP, Moore DE, Wright JK, Pinson CW. Reduction in corticosteroids is associated with better health-related quality of life after liver transplantation. J Am Coll Surg 2012;214:164-173.

10. Annema C, Roodbol PF, Stewart RE, Porte RJ, Ranchor AV. Prevalence of psychological problems and associated transplant-related variables at different time periods after liver transplantation. Liver Transpl 2015;21:524-538. 